\title{
JACOBI-LIKE FORMS AND POWER SERIES BUNDLES
}

\author{
MIN HO LEE
}

\begin{abstract}
Jacobi-like forms are certain formal power series which generalise Jacobi forms in some sense, and they are closely linked to modular forms when their coefficients are holomorphic functions on the Poincaré upper half plane. We construct two types of vector bundles whose fibres are isomorphic to the space of certain formal power series and whose sections can be identified with Jacobi-like forms for a discrete subgroup of $S L(2, \mathbb{R})$.
\end{abstract}

\section{INTRODUCTION}

Jacobi forms generalise classical theta functions, and they occur naturally in number theory, for example, as Fourier coefficients of Siegel modular forms. Various arithmetic aspects of Jacobi forms have been studied in numerous papers in recent years (see, for example $[1,2,4,6,9])$. Geometrically, they are also related to families of Abelian varieties or elliptic genera (see $[5,7,8,10]$ ). Jacobi-like forms generalise Jacobi forms in some sense, and they are certain formal power series satisfying a certain transformation formula relative to an action of a discrete subgroup $\Gamma$ of $S L(2, \mathbb{R})$. Since this transformation formula is essentially one of the two conditions that must be satisfied by Jacobi forms for $\Gamma$, Jacobi-like forms may be regarded as a generalisation of Jacobi forms. When their coefficients are holomorphic functions on the Poincaré upper half plane $\mathcal{H}$, Jacobi-like forms are closely linked to modular forms. Indeed, the transformation formula for Jacobilike forms for $\Gamma$ determines functional equations among their coefficients, which can be used to express those coefficients in terms of derivatives of a certain sequence of modular forms for $\Gamma$ (see $[3,11]$ ). Thus there is in fact a correspondence between Jacobi-like forms and sequences of modular forms. One of the applications of such a correspondence is the construction of bilinear differential operators on modular forms known as Rankin-Cohen brackets. Rankin-Cohen brackets can also be constructed using $\Gamma$-invariant pseudodifferential operators, and naturally there is a close relation between Jacobi-like forms and such pseudodifferential operators (see [3]).

It is well-known that modular forms for a discrete subgroup $\Gamma \subset S L(2, \mathbb{R})$ as above can be regarded as sections of a line bundle over the modular curve associated to $\Gamma$.

Received 21st March, 2002

Copyright Clearance Centre, Inc. Serial-fee code: 0004-9727/02 \$A2.00+0.00. 
Similarly, automorphic forms on a more general semisimple Lie group can also be interpreted as sections of a vector bundle over a locally symmetric space. It is also known that Jacobi forms can be interpreted as sections of certain vector bundles (see $[\mathbf{7}, \mathbf{8}, \mathbf{1 0}]$ ). In this paper we study similar interpretations for Jacobi-like forms. More specifically, we construct two types of vector bundles whose fibres are isomorphic to the space of certain formal power series and whose sections can be identified with Jacobi-like forms for $\Gamma$.

\section{JACOBI-LIKE FORMS}

In this section we review Jacobi-like forms for a discrete subgroup of $S L(2, \mathbb{R})$ whose coefficients are holomorphic functions on the Poincaré upper half plane and discuss some of their properties.

Let $\mathcal{H}$ be the Poincaré upper half plane on which $S L(2, \mathbb{R})$ acts as usual by linear fractional transformations. Thus, given $\gamma=\left(\begin{array}{ll}a & b \\ c & d\end{array}\right) \in S L(2, \mathbb{R})$ and $z \in \mathcal{H}$, we have

$$
\gamma z=\frac{a z+b}{c z+d}
$$

For such $\gamma$ and $z$, we set

$$
j(\gamma, z)=c z+d .
$$

Then we see easily that the resulting $\operatorname{map} j: S L(2, \mathbb{R}) \times \mathcal{H} \rightarrow \mathbb{C}$ is an automorphy factor, which means that it satisfies

$$
j\left(\gamma \gamma^{\prime}, z\right)=j\left(\gamma, \gamma^{\prime} z\right) j\left(\gamma^{\prime}, z\right)
$$

for all $\gamma, \gamma^{\prime} \in S L(2, \mathbb{R})$ and $z \in \mathcal{H}$.

Let $\mathcal{F}$ be the ring of holomorphic functions on $\mathcal{H}$, and let $\mathcal{F}[[X]]$ denote the set of formal power series in $X$ with coefficients in $\mathcal{F}$. Let $\mathbb{C}^{\times}=\mathbb{C}-\{0\}$ be the set of nonzero complex numbers, and set

$$
\mathbb{C}^{\times} X=\left\{\lambda X \mid \lambda \in \mathbb{C}^{\times}\right\}
$$

which can be identified with $\mathbb{C}^{\times}$. Using (2.2), we see that $S L(2, \mathbb{R})$ acts on $\mathcal{H} \times \mathbb{C}^{\times} X$ by

$$
\gamma \cdot(z, \lambda X)=\left(\gamma z, j(\gamma, z)^{-2} \lambda X\right)
$$

for all $z \in \mathcal{H}, \lambda \in \mathbb{C}^{\times}$and $\gamma \in S L(2, \mathbb{R})$. Throughout this paper we fix integers $\xi$ and $\eta$, and set

$$
J_{\xi, \eta}(\gamma,(z, \lambda X))=j(\gamma, z)^{\xi} \exp (c \lambda \eta X / j(\gamma, z)) \in \mathcal{F}[[X]]
$$

for $z \in \mathcal{H}, \lambda \in \mathbb{C}^{\times}$and $\gamma=\left(\begin{array}{ll}a & b \\ c & d\end{array}\right) \in S L(2, \mathbb{R})$. The next lemma shows that $J_{\xi, \eta}$ is an automorphy factor. 
LEMma 2.1. The map $J_{\xi, \eta}: S L(2, \mathbb{R}) \times\left(\mathcal{H} \times \mathbb{C}^{\times} X\right) \rightarrow \mathcal{F}[[X]]$ given by (2.4) satisfies

$$
J_{\xi, \eta}\left(\gamma \gamma^{\prime},(z, \lambda X)\right)=J_{\xi, \eta}\left(\gamma, \gamma^{\prime} \cdot(z, \lambda X)\right) J_{\xi, \eta}\left(\gamma^{\prime},(z, \lambda X)\right)
$$

for all $z \in \mathcal{H}, \lambda \in \mathbb{C}^{\times}$and $\gamma, \gamma^{\prime} \in S L(2, \mathbb{R})$, where $\gamma^{\prime} \cdot(z, \lambda X)$ is as in (2.3).

$$
\text { Proof: Let } \gamma=\left(\begin{array}{ll}
a & b \\
c & d
\end{array}\right), \gamma^{\prime}=\left(\begin{array}{ll}
a^{\prime} & b^{\prime} \\
c^{\prime} & d^{\prime}
\end{array}\right) \in S L(2, \mathbb{R}) \text {, so that }
$$

$$
\gamma \gamma^{\prime}=\left(\begin{array}{ll}
a a^{\prime}+b c^{\prime} & c a^{\prime}+d c^{\prime} \\
c a^{\prime}+d c^{\prime} & c b^{\prime}+d d^{\prime}
\end{array}\right) .
$$

Then, using (2.2), (2.3) and (2.4), for each $z \in \mathcal{H}$ and $\lambda \in \mathbb{C}^{\times}$we have

$$
\begin{aligned}
J_{\xi, \eta}\left(\gamma, \gamma^{\prime} \cdot(z, \lambda X)\right) J_{\xi, \eta}\left(\gamma^{\prime},(z, \lambda X)\right) & =j\left(\gamma, \gamma^{\prime} z\right)^{\xi} j\left(\gamma^{\prime}, z\right)^{\xi} \exp \left[\frac{c j\left(\gamma^{\prime}, z\right)^{-2} \lambda \eta X}{j\left(\gamma, \gamma^{\prime} z\right)}+\frac{c^{\prime} \lambda \eta X}{j\left(\gamma^{\prime}, z\right)}\right] \\
& =j\left(\gamma \gamma^{\prime}, z\right)^{\xi} \exp \left[\frac{\lambda \eta X}{j\left(\gamma \gamma^{\prime}, z\right)}\left(\frac{c}{j\left(\gamma^{\prime}, z\right)}+c^{\prime} j\left(\gamma, \gamma^{\prime} z\right)\right)\right] .
\end{aligned}
$$

However, by using the relation $a^{\prime} d^{\prime}-b^{\prime} c^{\prime}=1$ we see that

$$
\begin{aligned}
\frac{c}{j\left(\gamma^{\prime}, z\right)}+c^{\prime} j\left(\gamma, \gamma^{\prime} z\right) & =\frac{c}{c^{\prime} z+d^{\prime}}+c^{\prime}\left(c\left(\frac{a^{\prime} z+b^{\prime}}{c^{\prime} z+d^{\prime}}\right)+d\right) \\
& =\frac{c^{\prime}\left(c a^{\prime}+d c^{\prime}\right) z+c+c b^{\prime} c^{\prime}+d c^{\prime} d^{\prime}}{c^{\prime} z+d^{\prime}} \\
& =c a^{\prime}+d c^{\prime}+\frac{c-c\left(a^{\prime} d^{\prime}-b^{\prime} c^{\prime}\right)}{c^{\prime} z+d^{\prime}}=c a^{\prime}+d c^{\prime},
\end{aligned}
$$

which is the $(2,1)$-entry of $\gamma \gamma^{\prime}$ by $(2.5)$. Hence we obtain

$$
\begin{aligned}
\mathscr{J}_{\xi, \eta}\left(\gamma, \gamma^{\prime} \cdot(z, \lambda X)\right) J_{\xi, \eta}\left(\gamma^{\prime},(z, \lambda X)\right) & =j\left(\gamma \gamma^{\prime}, z\right)^{\xi} \exp \left[\frac{\lambda \eta\left(a^{\prime} c+c^{\prime} d\right) X}{j\left(\gamma \gamma^{\prime}, z\right)}\right] \\
& =J_{\xi, \eta}\left(\gamma \gamma^{\prime}, z\right),
\end{aligned}
$$

and therefore the lemma follows.

Let $\Gamma$ be a discrete subgroup of $S L(2, \mathbb{R})$. Then we can consider the associated Jacobi-like forms. Our definition will be slightly more general than the one in [11] because of the inclusion of the index $\eta$.

DEFINITION 2.2: A formal power series $\psi(z, X) \in \mathcal{F}[[X]]$ is a Jacobi-like form for $\Gamma$ of weight $\xi$ and index $\eta$ if it satisfies

$$
\psi(\gamma \cdot(z, X))=J_{\xi, \eta}(\gamma,(z, X)) \psi(z, X)
$$

for all $z \in \mathcal{H}$ and $\gamma \in \Gamma$. We denote by $\mathcal{J}_{\xi, \eta}(\Gamma)$ the space of all Jacobi-like forms for $\Gamma$ of weight $\xi$ and index $\eta$. 
REMARK 2.3. Jacobi-like forms generalise Jacobi forms in the following sense. By (2.3), (2.4) and (2.6) a power series $\psi(z, X) \in \mathcal{F}[[X]]$ is an element of $\mathcal{J}_{\xi, \eta}(\Gamma)$ if and only if

$$
\psi\left(\gamma z, j(\gamma, z)^{-2} X\right)=j(\gamma, z)^{\xi} \exp (c \eta X / j(\gamma, z)) \psi(z, X)
$$

for all $z \in \mathcal{H}$ and $\gamma=\left(\begin{array}{ll}a & b \\ c & d\end{array}\right) \in \Gamma$. Let $X=w^{2}$, and consider the formal power series $\Psi(z, w) \in \mathcal{F}\left[\left[w^{2}\right]\right]$ given by

$$
\Psi(z, w)=\psi\left(z, 2 \pi i w^{2}\right)
$$

Then, using (2.7), we obtain

$$
\begin{aligned}
\Psi\left(\gamma z, j(\gamma, z)^{-1} w\right) & =\psi\left(\gamma z, j(\gamma, z)^{-2} 2 \pi i w^{2}\right) \\
& =j(\gamma, z)^{\xi} \exp \left(2 \pi i c \eta w^{2} / j(\gamma, z)\right) \psi\left(z, 2 \pi i w^{2}\right) \\
& =j(\gamma, z)^{\xi} \exp \left(2 \pi i c \eta w^{2} / j(\gamma, z)\right) \Psi(z, w) .
\end{aligned}
$$

Thus we see that $\Psi(z, w)$ satisfies one of the two transformation formulas which define Jacobi forms for $\Gamma$ of weight $\xi$ and index $\eta$ (see [4, Chapter I]).

The next lemma describes the condition for a formal power series to be a Jacobi-like form in terms of its coefficient functions.

LEMMA 2.4. Let $\psi(z, X)=\sum_{k=u}^{\infty} f_{k}(z) X^{k} \in \mathcal{F}[[X]]$ for some nonnegative integer $u$. Then $\psi(z, X)$ is an element of $\mathcal{J}_{\xi, \eta}(\Gamma)$ if and only if

$$
f_{k}(\gamma z) j(\gamma, z)^{-2 k-\xi}=\sum_{r=0}^{k-u} \frac{1}{r !}\left(\frac{c \eta}{c z+d}\right)^{r} f_{k-r}(z)
$$

for all $z \in \mathcal{H}, g=\left(\begin{array}{ll}a & b \\ c & d\end{array}\right) \in \Gamma$, and $k \geqslant u$.

Proof: Given $z \in \mathcal{H}$ and $\gamma=\left(\begin{array}{ll}a & b \\ c & d\end{array}\right) \in \Gamma$, using (2.7), we see that $\Phi(z, X)$ is an element of $\mathcal{J}_{\xi, \eta}(\Gamma)$ if and only if

$$
\begin{aligned}
\sum_{k=u}^{\infty} f_{k}(\gamma z)(c z+d)^{-2 k-\xi} X^{k} & =\left(\sum_{r=0}^{\infty} \frac{1}{r !} \frac{(c \eta)^{r}}{(c z+d)^{r}} X^{r}\right)\left(\sum_{\ell=u}^{\infty} f_{\ell}(z) X^{\ell}\right) \\
& =\sum_{r=0}^{\infty} \sum_{\ell=u}^{\infty} \frac{1}{r !} \frac{(c \eta)^{r}}{(c z+d)^{r}} f_{\ell}(z) X^{r+\ell} \\
& =\sum_{k=u}^{\infty} \sum_{r=0}^{k-u} \frac{1}{r !} \frac{(c \eta)^{r}}{(c z+d)^{r}} f_{k-r}(z) X^{k}
\end{aligned}
$$

Hence we obtain (2.8) by comparing the coefficients of $X^{k}$. 
REMARK 2.5. The relation in (2.8) is a modified version of [3, Proposition 2, (3)] or [11, (15)]. If $\eta=1$, we can also generalise [3, Proposition $2,(5)]$ by showing that $\sum_{k=u}^{\infty} f_{k}(z) X^{k}$ is an element of $\mathcal{J}_{\xi, \eta}(\Gamma)$ if and only if

$$
f_{k}(z)=\sum_{r=0}^{k-u} \frac{\eta^{r}}{r !(2 k-r+\xi-1) !} f_{k-r}^{(r)}(z)
$$

for all $k \geqslant u$, where $f_{n} \in M_{2 n+\xi}(\Gamma)$ for all $n \geqslant u$.

\section{POWER SERIES BUNDLES OF THE FIRST TYPE}

In this section we construct a vector bundle over the quotient of the product $\mathcal{H} \times \mathbb{C}^{\times} X$ by a discrete subgroup of $S L(2, \mathbb{R})$ whose fibre is the space of certain formal power series in $X$ with constant coefficients and whose sections can be identified with Jacobi-like forms.

Let $\mathbb{C}[[X]]$ be the set of formal power series in $X$ with coefficients in $\mathbb{C}$. Let $u$ be a nonnegative integer, and denote by

$$
\mathbb{C}[[X]]_{u}=X^{u} \mathbb{C}[[X]]
$$

the subspace of $\mathbb{C}[[X]]$ consisting of formal power series of the form $\sum_{r=u}^{\infty} A_{r} X^{r}$ with $A_{r} \in \mathbb{C}$. Given $f(X) \in \mathbb{C}[[X]]_{u},(z, \lambda X) \in \mathcal{H} \times \mathbb{C}^{\times} X$ and $\gamma \in S L(2, \mathbb{R})$, we set

$$
\gamma \cdot((z, \lambda X), f(X))=\left(\gamma \cdot(z, \lambda X), J_{\xi, \eta}(\gamma,(z, \lambda X)) f(X)\right),
$$

where $\gamma \cdot(z, \lambda X)$ and $J_{\xi, \eta}(\gamma,(z, \lambda X))$ are as in (2.3) and (2.4), respectively. Then by Lemma 2.1 we see that the formula (3.2) determines an action of $S L(2, \mathbb{R})$ on the space $\left(\mathcal{H} \times \mathbb{C}^{\times} X\right) \times \mathbb{C}[[X]]_{u}$. Let $\Gamma$ be a discrete subgroup of $S L(2, \mathbb{R})$ as in Section 2, and set

$$
\mathcal{P}_{\xi, \eta}^{u}=\Gamma \backslash\left(\mathcal{H} \times \mathbb{C}^{\times} X\right) \times \mathbb{C}[[X]]_{u}, \quad W=\Gamma \backslash \mathcal{H} \times \mathbb{C}^{\times} X,
$$

where the quotient is taken with respect to the actions of $\Gamma$ given by (3.2) and (2.3), respectively. Then the natural projection map

$$
\left(\mathcal{H} \times \mathbb{C}^{\times} X\right) \times \mathbb{C}[[X]]_{u} \rightarrow \mathcal{H} \times \mathbb{C}^{\times} X
$$

induces a surjective map

$$
\pi: \mathcal{P}_{\xi, \eta}^{u} \rightarrow W
$$

such that $\pi^{-1}(w)$ is isomorphic to $\mathbb{C}[[X]]_{u}$ for each $w \in W$. Hence $\mathcal{P}_{\xi, \eta}^{u}$ can be regarded as a vector bundle over $W$ whose fibre is the space $\mathbb{C}[[X]]_{u}$ of formal power series in $X$. We denote by $\Gamma_{0}\left(W, \mathcal{P}_{\xi, \eta}^{u}\right)$ the space of holomorphic sections of this bundle, that is, the space of holomorphic maps $s: W \rightarrow \mathcal{P}_{\xi, \eta}^{u}$ such that $\pi \circ s=1_{W}$. 
REMARK 3.1. Given $\psi(z, X)=\sum_{n=u}^{\infty} a_{n}(z) X^{n} \in \mathcal{F}[[X]]$ with $u \geqslant 0$, we consider the associated map

$$
\tilde{\psi}: \mathcal{H} \times \mathbb{C}^{\times} X \rightarrow \mathbb{C}[[X]]_{u}
$$

defined by

$$
\widetilde{\psi}(z, \lambda X)=\sum_{n=0}^{\infty} a_{n}(z) \lambda^{n} X^{n}
$$

for all $z \in \mathcal{H}$ and $\lambda \in \mathbb{C}^{\times}$. Then we see easily that $\psi(z, X)$ is an element of $\mathcal{J}_{\xi, \eta}(\Gamma)$ if and only if

$$
\widetilde{\psi}(\gamma \cdot(z, \lambda X))=J_{\xi, \eta}(\gamma,(z, \lambda X)) \widetilde{\psi}(z, \lambda X)
$$

for all $z \in \mathcal{H}, \lambda \in \mathbb{C}^{\times}$and $\gamma \in \Gamma$.

THEOREM 3.2. The space $\Gamma_{0}\left(W, \mathcal{P}_{\xi, \eta}^{u}\right)$ of sections of the bundle $\mathcal{P}_{\xi, \eta}^{u}$ over $W$ is canonically isomorphic to the space $\mathcal{J}_{\xi, \eta}(\Gamma)$ of Jacobi-like forms for $\Gamma$ of weight $\xi$ and index $\eta$.

Proof: Let $s: W \rightarrow \mathcal{P}_{\xi, \eta}^{u}$ be a section of $\mathcal{P}_{\xi, \eta}^{u}$, and denote by $p: \mathcal{H} \times \mathbb{C}^{\times} X$ $\rightarrow W=\Gamma \backslash \mathcal{H} \times \mathbb{C}^{\times} X$ the natural projection map. Given $(z, \lambda X) \in \mathcal{H} \times \mathbb{C}^{\times} X$, we have

$$
s(p(z, \lambda X))=\left[\left((z, \lambda X), \mu_{(z, \lambda X)}\right)\right] \in \Gamma \backslash\left(\mathcal{H} \times \mathbb{C}^{\times} X\right) \times \mathbb{C}[[X]]_{u}
$$

for some element $\mu_{(z, \lambda X)} \in \mathbb{C}[[X]]_{u}$, where $[(\cdot)]$ denotes the $\Gamma$-orbit of $(\cdot)$. We define the $\operatorname{map} \psi_{s}: \mathcal{H} \times \mathbb{C}^{\times} X \rightarrow \mathbb{C}[[X]]_{u}$ by

$$
\psi_{s}(z, \lambda X)=\mu_{(z, \lambda X)}
$$

Given $\gamma \in \Gamma$, since $p(\gamma w)=p(w)$ for each $w \in \mathcal{H} \times \mathbb{C}^{\times} X$, we have

$$
s(p(z, \lambda X))=s(p(\gamma \cdot(z, \lambda X)))=\left[\left(\gamma \cdot(z, \lambda X), \mu_{\gamma \cdot(z, \lambda X)}\right)\right] .
$$

On the other hand, since $[(\cdot)]$ denotes the $\Gamma$-orbit, by (2.3), we obtain

$$
\begin{aligned}
s(p(z, \lambda X)) & =\left[\gamma \cdot\left((z, \lambda X), \mu_{(z, \lambda X)}\right)\right] \\
& =\left[\left(\gamma \cdot(z, \lambda X), J_{\xi, \eta}(\gamma,(z, \lambda X)) \mu_{(z, \lambda X)}\right)\right] .
\end{aligned}
$$

Comparing this with (3.4) and using (3.3), we see that

$$
\begin{aligned}
\psi_{s}(\gamma \cdot(z, \lambda X)) & =\mu_{\gamma \cdot(z, \lambda X)} \\
& =J_{\xi, \eta}(\gamma,(z, \lambda X)) \mu_{(z, \lambda X)} \\
& =J_{\xi, \eta}(\gamma,(z, \lambda X)) \psi_{s}(z, \lambda X)
\end{aligned}
$$


Thus, by Remark 3.1 the map $\psi_{s}$ may be regarded as a Jacobi-like form for $\Gamma$. Suppose now that $\psi$ is a Jacobi-like form for $\Gamma$, and let $\tilde{\psi}: \mathcal{H} \times \mathbb{C}^{\times} X \rightarrow \mathbb{C}[[X]]_{\boldsymbol{u}}$ be the associated map described in Remark 3.1. Then we have

$$
\widetilde{\psi}(\gamma \cdot(z, \lambda X))=J_{\xi, \eta}(\gamma,(z, \lambda X)) \widetilde{\psi}(z, \lambda X)
$$

for all $z \in \mathcal{H}$ and $\lambda \in \mathbb{C}$. We define the map $s_{\psi}: W \rightarrow \mathcal{P}_{\xi, \eta}^{u}$ by

$$
s_{\psi}(p(z, \lambda X))=[(z, \lambda X), \tilde{\psi}(\gamma(z, \lambda X))]
$$

for all $(z, \lambda X) \in \mathcal{H} \times \mathbb{C}^{\times} X$. Then, using (3.2) and (3.5), we have

$$
\begin{aligned}
s_{\psi}(p(\gamma(z, \lambda X))) & =[(\gamma(z, \lambda X), \widetilde{\psi}(\gamma(z, \lambda X)))] \\
& =\left[\left(\gamma(z, \lambda X), J_{\xi, \eta}(\gamma,(z, \lambda X)) \widetilde{\psi}(z, \lambda X)\right)\right] \\
& =[\gamma \cdot(z, \lambda X), \widetilde{\psi}(\gamma(z, \lambda X))] \\
& =[(z, \lambda X), \widetilde{\psi}(\gamma(z, \lambda X))]=s_{\psi}(p(z, \lambda X))
\end{aligned}
$$

hence $s_{\psi}$ is well-defined. Since clearly $\pi \circ s_{\psi}=1_{W}$, it follows that $s_{\psi}$ is an element of $\Gamma_{0}\left(W, \mathcal{P}_{\xi, \eta}^{u}\right)$.

\section{POWER SERIES BUNDLES OF THE SECOND TYPE}

In this section we construct another type of vector bundle whose fibre is the space of formal power series in $X$ with constant coefficients and whose sections can be identified with Jacobi-like forms. This bundle will be over the quotient of the Poincare upper half plane $\mathcal{H}$ by a discrete subgroup of $S L(2, \mathbb{R})$.

Let $\xi, \eta \in \mathbb{Z}$ and $\Gamma \subset S L(2, \mathbb{R})$ be as in Section 2. Given nonnegative integers $\ell$ and $r$, we set

$$
\Xi_{\ell, r}(\gamma, z)=\frac{(-c \eta)^{\ell}}{\ell ! j(\gamma, z)^{\ell+2 r+\xi}}
$$

for all $z \in \mathcal{H}$ and $\gamma=\left(\begin{array}{ll}a & b \\ c & d\end{array}\right) \in \Gamma$, where $j(\gamma, z)$ is as in (2.1). Then we see that

$$
\begin{aligned}
\sum_{\ell=0}^{\infty} \Xi_{\ell, r}(\gamma, z) X^{\ell} & =j(\gamma, z)^{-2 r-\xi} \sum_{\ell=0}^{\infty} \frac{1}{\ell !}\left(\frac{-c \eta X}{j(\gamma, z)}\right)^{\ell} \\
& =j(\gamma, z)^{-2 r-\xi} \exp (-c \eta X / j(\gamma, z)) \\
& =j(\gamma, z)^{-2 r} J_{\xi, \eta}(\gamma,(z, X))^{-1},
\end{aligned}
$$


where $J_{\xi, \eta}$ is as in (2.4). Given $f(X)=\sum_{r=u}^{\infty} A_{r} X^{r} \in \mathbb{C}[[X]]_{u}$ for some $u \geqslant 0$ with $\mathbb{C}[[X]]_{u}$ being as in (3.1), we set

$$
\gamma \cdot(z, f(X))=\left(\gamma z, \sum_{r=u}^{\infty} \sum_{\ell=0}^{\infty} \Xi_{\ell, r}\left(\gamma^{-1}, \gamma z\right) A_{r} X^{\ell+r}\right)
$$

for all $\gamma \in \Gamma$ and $z \in \mathcal{H}$.

PROPOSITION 4.1. The formula (4.3) determines an action of $\Gamma$ on the space $\mathcal{H} \times \mathbb{C}[[X]]_{u}$.

Proof: Let $\gamma, \gamma^{\prime} \in \Gamma, z \in \mathcal{H}$, and $f(X)=\sum_{r=u}^{\infty} A_{r} X^{r} \in \mathbb{C}[[X]]_{u}$. Using (4.1), (4.2) and (4.3), we see that

$$
\begin{aligned}
& \gamma^{\prime} \cdot(\gamma \cdot(z, f(X)))=\gamma^{\prime} \cdot\left(\gamma z, \sum_{r=u}^{\infty} \sum_{\ell=0}^{\infty} \Xi_{\ell, r}\left(\gamma^{-1}, \gamma z\right) A_{r} X^{\ell+r}\right) \\
&=\left(\gamma^{\prime} \gamma z, \sum_{r=u}^{\infty} \sum_{\ell=0}^{\infty} \sum_{m=0}^{\infty} \Xi_{m, \ell+r}\left(\gamma^{\prime-1}, \gamma^{\prime} \gamma z\right) \Xi_{\ell, r}\left(\gamma^{-1}, \gamma z\right) A_{r} X^{m+\ell+r}\right) \\
&=\left(\gamma^{\prime} \gamma z, \sum_{r=u}^{\infty} A_{r} X^{r} \sum_{\ell=0}^{\infty} \Xi_{\ell, r}\left(\gamma^{-1}, \gamma z\right) X^{\ell} \sum_{m=0}^{\infty} \Xi_{m, \ell+r}\left(\gamma^{\prime-1}, \gamma^{\prime} \gamma z\right) X^{m}\right) \\
&=\left(\gamma^{\prime} \gamma z, \sum_{r=u}^{\infty} A_{r} X^{r} \sum_{\ell=0}^{\infty} \Xi_{\ell, r}\left(\gamma^{-1}, \gamma z\right)\left(j\left(\gamma^{\prime-1}, \gamma^{\prime} \gamma z\right)^{-2} X\right)^{\ell}\right. \\
&=\left(\gamma^{\prime} \gamma z, \sum_{r=u}^{\infty} A_{r} X^{r} j\left(\gamma^{-1}, \gamma z\right)^{-2 \tau} J_{\xi, \eta}\left(\gamma^{-1},\left(\gamma z, j\left(\gamma^{\prime-1}, \gamma^{\prime} \gamma z\right)^{-2} X\right)\right)^{-1}\right. \\
&\left.\times j\left(\gamma^{\prime-1}, \gamma^{\prime} \gamma z\right)^{-2 r} J_{\xi, \eta}\left(\gamma^{\prime-1},\left(\gamma^{\prime} \gamma z, X\right)\right)^{-1}\right) .
\end{aligned}
$$

However, we have

$$
j\left(\left(\gamma \gamma^{\prime}\right)^{-1}, \gamma^{\prime} \gamma z\right)=j\left(\gamma^{-1} \gamma^{-1}, \gamma^{\prime} \gamma z\right)=j\left(\gamma^{-1}, \gamma z\right) j\left(\gamma^{\prime-1}, \gamma^{\prime} \gamma z\right)
$$

and using Lemma 2.1,

$$
\begin{aligned}
J_{\xi, \eta}\left(\gamma^{-1},(\gamma z, j\right. & \left.\left(\gamma^{-1}, \gamma^{\prime} \gamma z\right)^{-2} X\right) J_{\xi, \eta}\left(\gamma^{-1},\left(\gamma^{\prime} \gamma z, X\right)\right) \\
& =J_{\xi, \eta}\left(\gamma^{-1}, \gamma^{-1} \cdot\left(\gamma^{\prime} \gamma z, X\right)\right) J_{\xi, \eta}\left(\gamma^{\prime-1},\left(\gamma^{\prime} \gamma z, X\right)\right) \\
& =J_{\xi, \eta}\left(\left(\gamma^{\prime} \gamma\right)^{-1},\left(\gamma^{\prime} \gamma z, X\right)\right) .
\end{aligned}
$$


Thus we obtain

$$
\begin{aligned}
\gamma^{\prime} \cdot(\gamma \cdot(z, f(X))) & =\left(\gamma^{\prime} \gamma z, \sum_{r=u}^{\infty} j\left(\left(\gamma^{\prime} \gamma\right)^{-1}, \gamma z\right)^{-2 r} J_{\xi, \eta}\left(\left(\gamma^{\prime} \gamma\right)^{-1},\left(\gamma^{\prime} \gamma z, X\right)\right)^{-1} A_{r} X^{r}\right) \\
& =\left(\gamma^{\prime} \gamma z, \sum_{r=u}^{\infty} \sum_{\ell=0}^{\infty} \Xi_{\ell, r}\left(\left(\gamma^{\prime} \gamma\right)^{-1}, \gamma^{\prime} \gamma z\right) A_{r} X^{\ell+r}\right) \\
& =\left(\gamma^{\prime} \gamma\right) \cdot(z, f(X))
\end{aligned}
$$

hence the proposition follows.

We denote the quotient of the space $\mathcal{H} \times \mathbb{C}[[X]]_{u}$ by $\Gamma$ with respect to the action shown in Proposition 4.1 by

$$
\mathcal{Q}_{\xi, \eta}^{u}=\Gamma \backslash \mathcal{H} \times \mathbb{C}[[X]]_{u},
$$

and set $U=\Gamma \backslash \mathcal{H}$. Then the natural projection map $\mathcal{H} \times \mathbb{C}[[X]]_{u} \rightarrow \mathcal{H}$ induces a surjective map $\varpi: \mathcal{Q}_{\xi, \eta}^{u} \rightarrow U$ such that $\varpi^{-1}(x)$ is isomorphic to $\mathbb{C}[[X]]_{u}$ for each $x \in U$. Thus $\mathcal{Q}_{\xi, \eta}^{u}$ has the structure of a complex vector bundle over $U$ whose fibre is the complex vector space $\mathbb{C}[[X]]_{u}$ of formal power series in $X$. We denote by $\Gamma_{0}\left(U, \mathcal{Q}_{\xi, \eta}^{u}\right)$ the space of all holomorphic sections of $\mathcal{Q}_{\xi, \eta}^{u}$ over $U$.

THEOREM 4.2. The space $\Gamma_{0}\left(U, \mathcal{Q}_{\xi, \eta}^{u}\right)$ of holomorphic sections of $\mathcal{Q}_{\xi, \eta}^{u}$ over $U=\Gamma \backslash \mathcal{H}$ is canonically isomorphic to the space $\mathcal{J}_{\xi, \eta}(\Gamma)$ of all Jacobi-like forms for $\Gamma$ of weight $\xi$ and index $\eta$.

ProOF: Let $\sigma: U \rightarrow \mathcal{Q}_{\xi, \eta}^{u}$ be a section of $\mathcal{Q}_{\xi, \eta}^{u}$ over $U$, and denote by $q: \mathcal{H} \rightarrow U$ the natural projection map. Given $z \in \mathcal{H}$, we have

$$
\sigma(q(z))=\left[\left(z, \sum_{k=u}^{\infty} A_{k, z} X^{k}\right)\right] \in \Gamma \backslash \mathcal{H} \times \mathbb{C}[[X]]_{u}
$$

for some sequence $\left(A_{k, z}\right)_{k=u}^{\infty}$ of complex numbers, where $[(\cdot)]$ denotes the $\Gamma$-orbit of the element $(\cdot)$ of $\mathcal{H} \times \mathbb{C}[[X]]_{u}$. We define the sequence $\left(f_{k}^{\sigma}(z)\right)_{k=u}^{\infty}$ of $\mathbb{C}$-valued functions on $\mathcal{H}$ by

$$
f_{k}^{\sigma}(z)=A_{k, z}
$$

for all $z \in \mathcal{H}$ and $k \geqslant u$. Let $\gamma=\left(\begin{array}{ll}a & b \\ c & d\end{array}\right) \in \Gamma$. Since the $\Gamma$-orbits of $z$ and $\gamma z$ are the same for each $z \in \mathcal{H}$, we have

$$
\sigma(q(z))=\sigma(q(\gamma z))=\left[\left(\gamma z, \sum_{k=u}^{\infty} A_{k, \gamma z} X^{k}\right)\right]
$$


On the other hand, since $[\gamma w]=[w]$ for each $w \in \mathcal{H} \times \mathbb{C}[[X]]_{u}$, by using (4.3) we have

$$
\begin{aligned}
\sigma(q(z)) & =\left[\gamma \cdot\left(z, \sum_{k=\imath}^{\infty} A_{k, z} X^{k}\right)\right] \\
& =\left[\left(\gamma z, \sum_{r=u}^{\infty} \sum_{\ell=0}^{\infty} \Xi_{\ell, r}\left(\gamma^{-1}, \gamma z\right) A_{r, z} X^{\ell+r}\right)\right] \\
& =\left[\left(\gamma z, \sum_{k=u}^{\infty} \sum_{\ell=0}^{k-u} \Xi_{\ell, k-\ell}\left(\gamma^{-1}, \gamma z\right) A_{k-\ell, z} X^{k}\right)\right]
\end{aligned}
$$

By comparing this with (4.5) and by using (4.1) and (4.4) we see that

$$
\begin{aligned}
f_{k}^{\sigma}(\gamma z)=A_{k, \gamma z} & =\sum_{\ell=0}^{k-u} \Xi_{\ell, k-\ell}\left(\gamma^{-1}, \gamma z\right) A_{k-\ell, z} \\
& =\sum_{\ell=0}^{k-u} \Xi_{\ell, k-\ell}\left(\gamma^{-1}, \gamma z\right) f_{k-\ell}^{\sigma}(z) \\
& =\sum_{\ell=0}^{k-u} \frac{(c \eta)^{\ell}}{\ell !} j\left(\gamma^{-1}, \gamma z\right)^{-2 k+\ell-\xi} f_{k-\ell}^{\sigma}(z)
\end{aligned}
$$

where we used the fact that the $(2,1)$-entry of $\gamma^{-1}$ is $(-c)$. However, by $(2.2)$ we have

$$
1=j\left(\gamma^{-1} \gamma, z\right)=j\left(\gamma^{-1}, \gamma z\right) j(\gamma, z)
$$

Thus we obtain

$$
f_{k}^{\sigma}(\gamma z)=\sum_{\ell=0}^{k-u} \frac{1}{\ell !}\left(\frac{c \eta}{j(\gamma, z)}\right)^{\ell} j(\gamma, z)^{2 k+\xi} f_{k-\ell}^{\sigma}(z)
$$

From this and Lemma 2.4 it follows that the formal power series $\sum_{k=0}^{\infty} f_{k}^{\sigma}(z) X^{k}$ is an element of $\mathcal{J}_{\xi, \eta}(\Gamma)$. On the other hand, suppose that $\psi(z, X)=\sum_{k=0}^{\infty} f_{k}(z) X^{k}$ is an element of $\mathcal{J}_{\xi, \eta}(\Gamma)$. We define the map $\sigma_{\psi}: U \rightarrow \mathcal{Q}_{\xi, \eta}^{u}$ by

$$
\sigma_{\psi}(q(z))=\left[\left(z, \sum_{k=u}^{\infty} f_{k}(z) X^{k}\right)\right]
$$

for all $z \in \mathcal{H}$. Then for each $\gamma \in \Gamma$, we have

$$
\begin{aligned}
\sigma_{\psi}(q(\gamma z)) & =\left[\left(\gamma z, \sum_{k=u}^{\infty} \sum_{\ell=0}^{k-u} \frac{(-c \eta)^{\ell}}{\ell !} j\left(\gamma^{-1}, \gamma z\right)^{-2 k+\ell-\xi} f_{k-\ell}(z) X^{k}\right)\right] \\
& =\left[\left(\gamma z, \sum_{k=u}^{\infty} \sum_{\ell=0}^{k-u} \Xi_{\ell, k-\ell, \xi}(\gamma, z) f_{k-\ell}(z) X^{k}\right)\right] \\
& =\left[\gamma \cdot\left(z, \sum_{k=u}^{\infty} f_{k}(z) X^{k}\right)\right] \\
& =\sigma_{\psi}(q(z))
\end{aligned}
$$


and therefore $\sigma_{\psi}$ is well-defined. Since clearly $\varpi \circ \sigma_{\psi}=1_{U}$, it follows that $\sigma_{\psi}$ is a holomorphic section of $\mathcal{Q}_{\xi, \eta}^{u}$ over $U$; hence the proof of the theorem is complete.

\section{REFERENCES}

[1] R. Berndt and R. Schmidt, Elements of the representation theory of the Jacobi group (Birkhäuser, Boston, 1998).

[2] R. Borcherds, 'Automorphic forms on $O_{s+2,2}(\mathbf{R})$ and infinite products', Invent. Math. 120 (1995), 161-213.

[3] P. Cohen, Y. Manin and D. Zagier, 'Automorphic pseudodifferential operators', in Algebraic aspects of nonlinear systems (Birkhäuser, Boston, 1997), pp. 17-47.

[4] M. Eichler and D. Zagier, The theory of Jacobi forms, Progress in Math. 55 (Birkhäuser, Boston, 1985).

[5] V. Gritsenko, 'Elliptic genus of Calabi-Yau manifolds and Jacobi and Siegel modular forms', St. Petersburg Math. J. 11 (2000), 781-804.

[6] W. Kohnen, 'Jacobi forms and Siegel modular forms: Recent results and problems', Enseign. Math. 39 (1993), 121-136.

[7] J. Kramer, 'A geometric approach to the theory of Jacobi forms', Compositio Math. 79 (1991), 1-19.

[8] M. H. Lee, 'Jacobi forms on symmetric domains and torus bundles over abelian schemes', J. Lie Theory 11 (2001), 545-557.

[9] N. Skoruppa, 'Developments in the theory of Jacobi forms', in Conference on automorphic forms and their applications. Khabarovsk 1988 (Inst. Appl. Math., Khabarovsk, 1990), pp. $167-185$.

[10] K. Wirthmiller, 'Root systems and Jacobi forms', Compositio Math. 82 (1992), 293-354.

[11] D. Zagier, 'Modular forms and differential operators', Proc. Indian Acad. Sci. Math. Sci. 104 (1994), 57-75.

Department of Mathematics

University of Northern Iowa

Cedar Falls, IA 50614

United States of America

e-mail: lee@math.uni.edu 